\title{
Together or separately? Evaluating the Content Free Period in PRIME using SimPRIME
}

\author{
Javier Matanza Domingo \\ Sadot Alexandres Fernández \\ Concepción Mora de Amarillas \\ Institute for Research in Technology (IIT) \\ ICAI School of Engineering \\ Comillas Pontifical University \\ Madrid, Spain \\ Email: jmatanza@comillas.edu
}

\author{
Gregorio López López \\ Polytechnic University of Madrid \\ Madrid, Spain \\ Email: gregorio.lopez.lopez@upm.es
}

\author{
Jose Ignacio Moreno \\ Carlos III University \\ Madrid, Spain \\ Email: joseignacio.moreno@uc3m.es
}

\begin{abstract}
PoweRline Intelligent Metering Evolution (PRIME) is a Narrowband Powerline Communication (NB-PLC) technology widely deployed in the last mile of Advanced Metering Infrastructure (AMI) in Spain (Iberdrola, Union Fenosa), which is also quickly spreading globally. The present paper addresses the evaluation of the two medium access control mechanisms used in this technology: the Shared Contention Period (SCP), where all nodes content to access the channel; and the Contention Free Period (CFP), where time slots are reserved in order to guarantee some transmission opportunities. Whereas the SCP is the default behavior given its simplicity, the use of the CFP may be necessary when using applications that require a given level of Quality of Service $(\mathrm{QoS})$. The paper first describes how the CFP has been implemented according to the standard in the PRIME network simulator SimPRIME. Moreover, the paper asseses how combining different number of nodes in the SCP and CFP affects the network performance in a series of illustrative scenarios. Results from simulations point out that, when all nodes transmit together in the SCP, it yields better network performance than using the CFP in general; and especially in highly populated networks. However, the paper also highlights trade-off solutions that allow guaranteeing QoS to certain nodes without compromising the performance of the rest of the network. Additionally, the paper proposes an amendment to the standard to increase the number of nodes that can use the CFP under the previous assumption.
\end{abstract}

Keywords-AMI, CFP, ITU-T G.9904, OMNeT++, PLC, PRIME, QoS, SCP, simPRIME, Smart Grid

\section{INTRODUCTION}

Advanced Metering Infrastructures (AMI) are being widely deployed worldwide and, specially, at European level [1], [2]. As a matter of fact, the European Commission estimates an accumulated investment of around 3500 million euros associated to the deployment of 195 million smart meters by 2020 only in Europe [3].

Narrowband Powerline Communication (NB-PLC) technologies are being widely used in the last mile of such AMI due to the many benefits they present from the Distribution System Operators' (DSO) perspective, such as the fact that they use the low voltage cable as communications medium, thus dramatically reducing deployment costs [4], [5].
PoweRline Intelligent Metering Evolution (PRIME) is a promising NB-PLC technology. Currently there are more than 13 million PRIME-compliant smart meters deployed worldwide [6] and by 2018 there will be around 15 million only in Spain, due to local regulation. PRIME was initially developed by leading DSO and chipset manufacturers, such as Iberdrola, Gas Natural Fenosa, Texas Instruments or ADD (now Microchip), and in 2012 its physical and data link layers were standardized by ITU-T [7].

Although PRIME is currently used mainly for billing purposes, there is a kind of research trend which focuses on the possibilities of delivering services or applications with more challenging communications requirements on top of this technology [8]-[10].

This paper elaborates on such a research trend. To be more precise, the paper focuses on the MAC sublayer of PRIME. At this sublayer, the standard defines both a Shared Contention Period (SCP) and a Contention Free Period (CFP), but currently only the SCP is implemented, despite the CFP may be useful to provide certain level of Quality-of-Service (QoS).

Therefore, the main goal of this paper is to evaluate the impact of using the CFP both on the overall performance of PRIME networks and on the performance of their individual nodes, taking the latency as performance metric. In order to achieve this goal, the CFP has been implemented, as defined in the standard, in the PRIME network simulator SimPRIME [11], developed as part of the PhD thesis of Prof. Dr. Javier Matanza [12].

The remainder of the paper is structured as follows. In section II, PRIME technology is summarized paying special attention to the MAC mechanisms related to this work. Section III describes how the CFP has been implemented in SimPRIME. Section IV analyzes the results obtained after simulating the use of the CFP in a series of representative scenarios. Finally, section V draws conclusions and future work. 


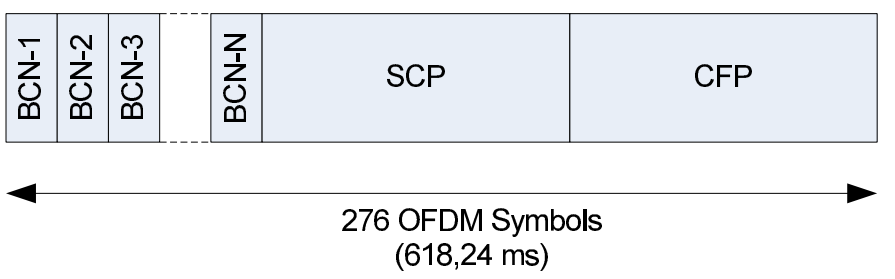

Fig. 1: Scheme of a PRIME' super-frame.

\section{OVERVIEW OF PRIME TECHNOLOGY}

PRIME is a second generation NB-PLC technology whose specification has been led by the PRIME Alliance and that has become an ITU-T standard [7]. The standard specifies the two lowest layers of the protocol stack, namely: physical (PHY) and data link layer. The data link layer comprises in turn two sublayers, namely: Medium Access Control (MAC) and a convergence layer which deals with multiplexing upper layer protocols, as well as with flow control and Automatic Repeat Request (ARQ) mechanisms.

At PHY level, PRIME can operate at the CENELEC-A $(41-89 \mathrm{kHz})$ or FCC $(155 \mathrm{kHz}-487 \mathrm{kHz})$ using an Orthogonal Frequency Division Multiplexing (OFDM) scheme with adaptive differential digital modulation, achieving a maximum data bit rate of up to $130 \mathrm{kbps}$, in the CENELEC-A band, and up to $1 \mathrm{Mbps}$, in the FCC band. More information regarding the PHY layer of PRIME or its performance can be found in the literature.

At MAC level, two main type of nodes are defined: Base Node (BN) and Service Node (SN). The Base Node represents the network coordinator, where the Service Nodes play the role of network slaves. In addition, the SNs, upon request to the $\mathrm{BN}$, can change their behaviour to Switch (SW). Switches are used as relays by the $\mathrm{BN}$ in order to increase its effective range. They are an important actor in PRIME networks and its position plays an important role in their performance, as studied in [13], [14].

Regarding the medium access mechanism, PRIME defines two types of access: a mandatory Contention Free Period (CFP) and an optional Shared Contention Period (SCP). Their main difference is that, while the former guarantees a time slot for SNs or BN to use the channel, in the latter, everybody is allowed to content for the channel and no access is guaranteed. Prior the SCP, a small period of time is reserved for beacon transmission, which is intended to broadcast basic network information and to provide synchronization. This super-frame structure lasts 276 OFDM symbols, as shown in Figure 1, and is periodically repeated over time.

Each of the previously defined access method has clear advantages and disadvantages. On one hand, the use of the $\mathrm{SCP}$ requires a lower network management traffic, which is basically reduced to the signal of the start and end of the period. On the other hand, using the SCP does not guarantee any QoS and channel access depends on network congestion. In case of using the CFP, thanks to the reservation of time slot every super-frame, channel access is guaranteed. However, an extra management traffic is required to indicate all nodes in the network when CFP slots are placed, so that none else transmit

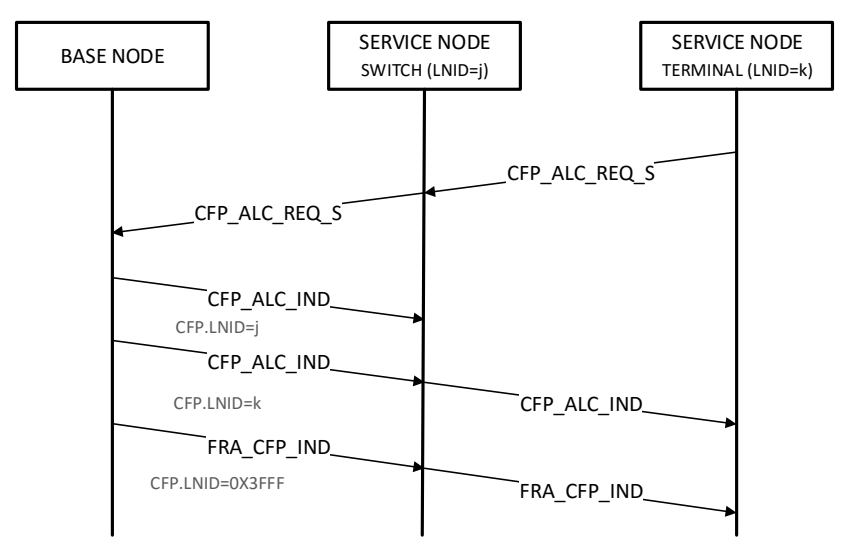

Fig. 2: Dialog for slot assignment during CFP.

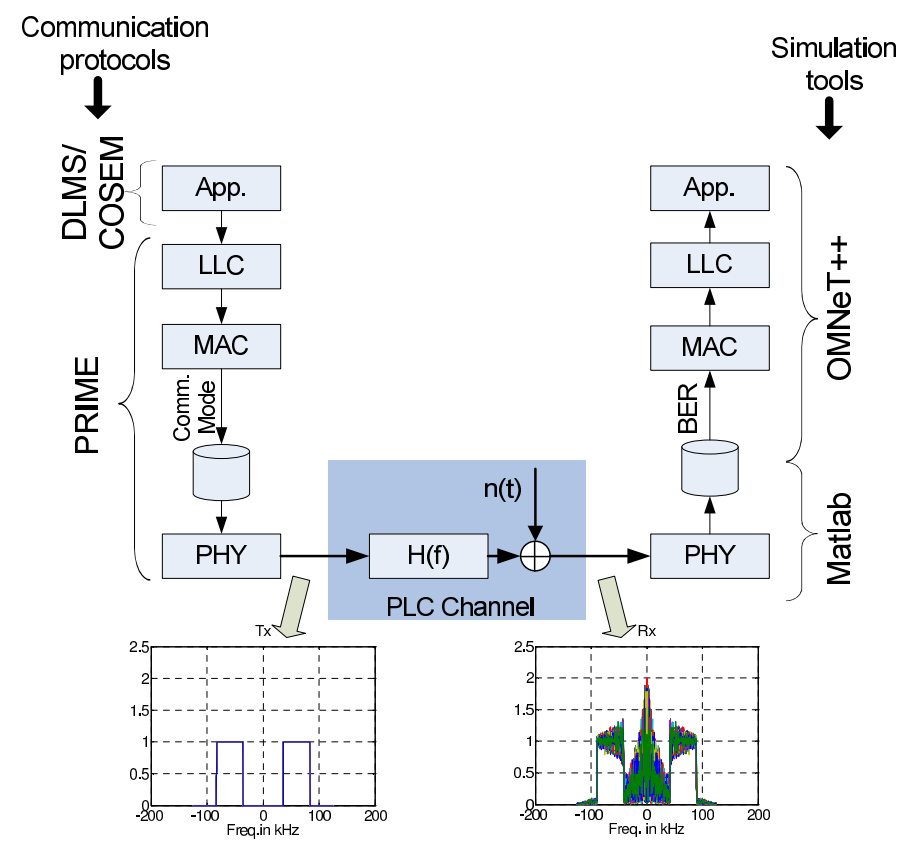

Fig. 3: Functional architecture of SimPRIME.

during these periods of time.

However, although these advantages and disadvantages are qualitative clear, they cannot be easily extrapolated to a quantitative network performance metric. This is in fact the main motivation for the present paper, where we provide an analysis via simulation of how the use of a combination of this two methods affects a PRIME network globally and individually.

\section{DEVElopMEnt Description}

In order to evaluate the effect of the SCP and CFP on the performance of PRIME networks, a simulation development has been carried out using a PRIME network simulator: SimPRIME [11]. SimPRIME combines MATLAB and OMNeT++ simulator [15] in order to model and simulate physical and upper layer effects respectively, as it is shown in Figure 3. 


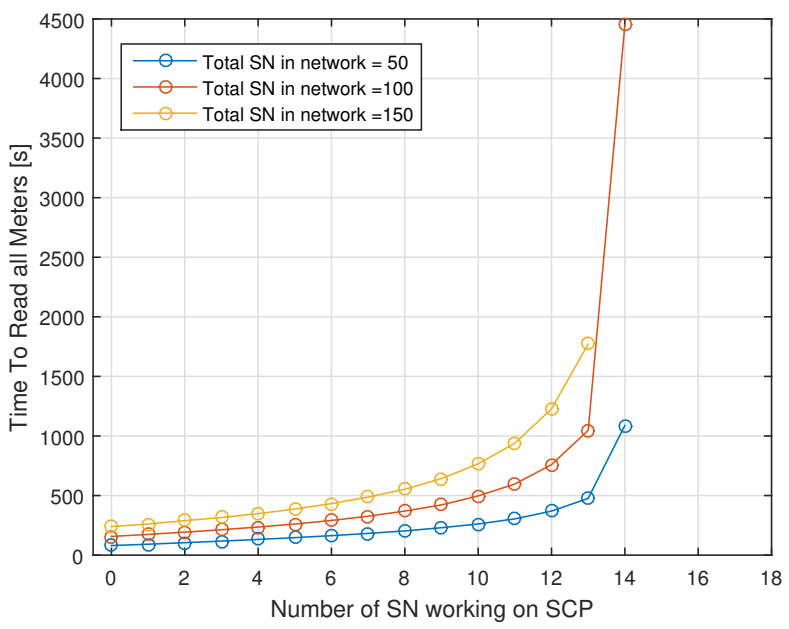

Fig. 4: Time to read all nodes in the network as a function of the number of $\mathrm{SN}$ in the CFP.

Even though SimPRIME has been presented and used in previous studies [9], [12]-[14], [16]-[18], this papers introduces the developments carried out in order to implement the CFP feature as described in PRIME standard. More specifically, the simulation environment has been extended with the dialog shown in Figure 2.

Figure 2 shows the dialog between a $\mathrm{BN}$ and a $\mathrm{SN}$ when the latter request for a CFP slot of a given duration to transmit data. Should the BN accept the request, it reserves both a slot for the $\mathrm{SN}$ to transmit data to the $\mathrm{BN}$ and a slot for the $\mathrm{BN}$ to transmit data to the SN (e.g., ACK). Additionally, if the $\mathrm{SN}$ were not directly registered to the $\mathrm{BN}$ but through a $\mathrm{SW}$, slots must be reserved for the SW in the up-link and down-link direction. Figure 2 also illustrates this last case, shwoing that both SW and SN are notified with the acceptance of the slot with a CLP_ALC_IND message. In addition to this message, a FRA_CFP_IND message is broadcast to all nodes in the network to inform of the new structure of the super-frame (recall Figure 1.

According to the standard, the maximum number of slots that can be reserved per super-trame is 32, which means that only $16 \mathrm{SN}$ can use the CFP, since half of them are required for the down-link communication (BN to $\mathrm{SN}$ ). The standard, however, does not limit the requested duration of those slots. The present study uses CFP slots corresponding to 376 bits and 256 bits for the $\mathrm{SN}$ and $\mathrm{BN}$ respectively. In terms of time, the slots represent $24 \mathrm{~ms}$ (or 10 OFDM symbols) available for the $\mathrm{SN}$ to transmit and $16.8 \mathrm{~ms}$ (or 7 OFDM symbols) available for the BN to transmit. This message lengths are coherent with other related studies, such as [8], [19].

\section{Simulation Results}

Using the simulation environment described in section III, a series of experiments have been run in order to evaluate the effect of using the CFP in the overall performance of PRIME networks as well as in the disaggregated performance of their nodes.
The scenario under study considers a low voltage network composed of a BN and a variable number of SN equidistantly distributed along the low voltage line. In order to avoid introducing too many variables in the study, the distance between $\mathrm{SN}$ is fixed in such a way that all the SN can communicate directly with the $\mathrm{BN}$, so there are no $\mathrm{SW}$ in the considered PRIME network.

As it has already been mentioned, latency is used as evaluation metric. To be more precise, two figures-of-merit are considered:

1) The time required by the $\mathrm{BN}$ to obtain a consumption measurement from all the SN in the network, computed as the time difference between the first request from the $\mathrm{BN}$ and the end of the response from the last SN. This parameter will be referred as the Time to Read All (TTRALL).

2) The time required by the $\mathrm{BN}$ to obtain a consumption measure from a single $\mathrm{SN}$, computed as the time difference between the request from the $\mathrm{BN}$ and the end of the response from the SN. This parameter will be referred as the Round Trip Time (RTT).

These figures-of-merit are selected because of their importance to DSO, since they provide them with an idea of the maximum allowed sampling frequency of the consumption data in each scenario. Each experiment is run 100 times in order to compute meaningful statistics of such figures-of-merit.

Figure 4 shows the median of the TTRAll as a function of the number of SN in the CFP and of the total number of SN in the network. Looking at each of the graphs individually, it can be seen that the TTRAll increases when the number of $\mathrm{SN}$ in the CFP increases, being specially remarkable when the number of $\mathrm{SN}$ in the CFP is close to the maximum (i.e., 16, c.f. section III). Comparing the three graphs, it can be observed that: (1) the higher the number of SN, the higher the TTRAll, and (2) the higher the number of SN, the lower the number of $\mathrm{SN}$ in the CFP required to make the degradation of the overall performance of the network remarkable, up to the point that in the scenario with $150 \mathrm{SN}$ and $14 \mathrm{SN}$ transmitting data in the CFP, the $\mathrm{BN}$ is not able to gather data from all the $\mathrm{SN}$ in the network (thus, the TTRAll would be infinite, which is why this point does not appear in Figure 4). This makes sense since, despite the latency of the SN in the CFP keeps constant, the duration of the SCP decreases when the number of $\mathrm{SN}$ in the CFP increases, so the higher the total number of $\mathrm{SN}$, the more difficult it will be to find the channel available in the SCP.

The compensation to the degradation of the overall network performance is that the SN transmitting in the CFP are guaranteed a certain level of QoS. In order to make this more evident, Figure 5 shows the median of the RTT for the SN transmitting both in the SCP and in the CFP as a function of the number of $\mathrm{SN}$ in the CFP. In this case, the scenarios with $100 \mathrm{SN}$ and $150 \mathrm{SN}$ have been selected as for being more problematic than the scenario with $50 \mathrm{SN}$. In Figure 5, it can be observed that the RTT for the SN transmitting in the CFP keeps indeed constant regardless the number of SN in the CFP and the total number of $\mathrm{SN}$ in the network, i.e., the use of the CFP indeed guarantees a certain level of QoS regardless the congestion of the network. It is worthwhile to remark upon 


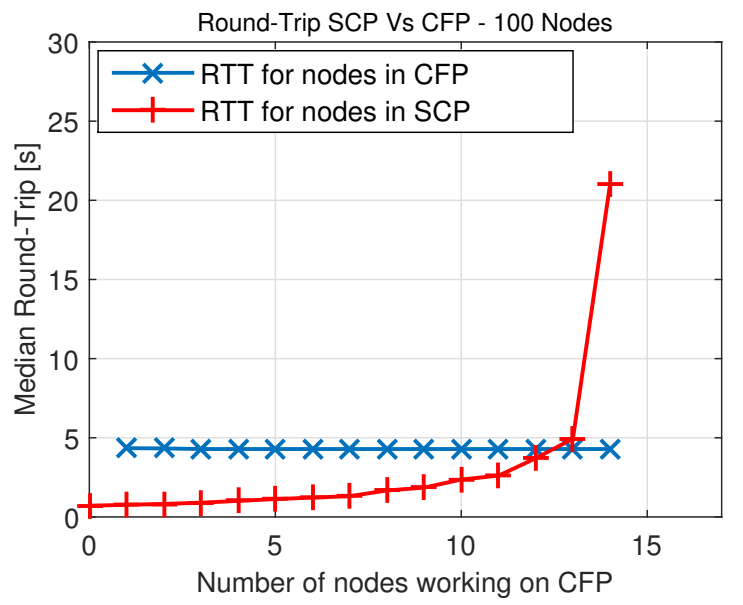

(a)

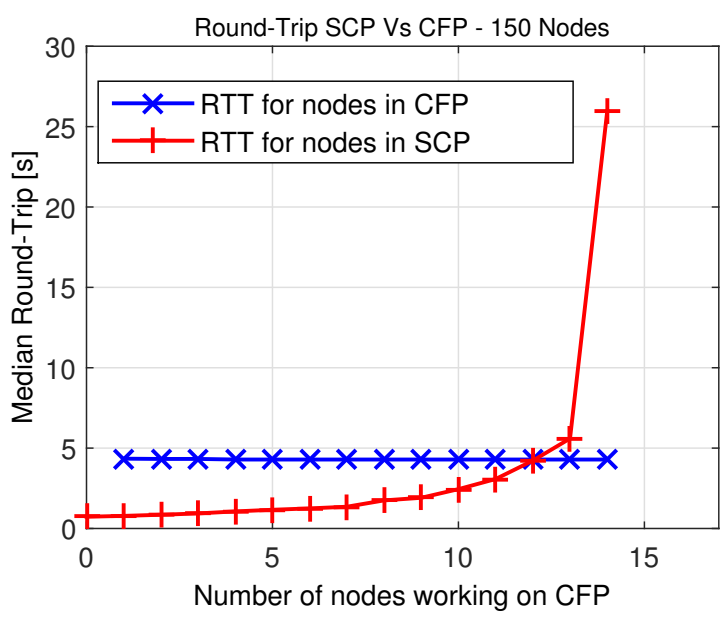

(b)

Fig. 5: Averaged RTT for nodes on CFP and SCP for different number of service nodes in the network.

the fact that the RTT for the SN in the CFP is constant but higher than the RTT for the SN in the SCP until the number of SN in the CFP gets close to the maximum. This is because the RTT in the CFP is guaranteed but also limited by the duration of the PRIME super-frame (276 OFDM symbols). It is also worthwhile to remark upon the fact that such an even condition (i.e., the RTT of the SN transmitting in the SCP is equal to the RTT of the SN transmitting in the CFP) seems to depend on the total number of SN in the network (notably, the higher the total number of $\mathrm{SN}$, the lower the number of $\mathrm{SN}$ in the CFP), as it can be observed in Figure 5 where this even condition happens for $13 \mathrm{SN}$ in the CFP when there are $100 \mathrm{SN}$ in the network (Figure 5 (a)) and for $12 \mathrm{SN}$ in the CFP when there are $150 \mathrm{SN}$ in the network (Figure 5 (b)). These results point out that it will be possible to guarantee a certain level of QoS to a given number of SN without compromising the overall network performance, such a number of safeguarded $\mathrm{SN}$ decreasing when the total of SN in the network increases.

Finally, Figure 6 allows to evaluate more in detail the actual quality of the communications in terms of the RTT depending on the total number of $\mathrm{SN}$ and the number of $\mathrm{SN}$ transmitting in the CFP. In this case, scenarios ranging from kind of best case (i.e., $50 \mathrm{SN}$ and $4 \mathrm{SN}$ transmitting in the CFP) to worst case (i.e., $100 \mathrm{SN}$ and $14 \mathrm{SN}$ transmitting in the CFP) are considered in order to better illustrate the difference in the individual communications performance. Thus, in Figure 5 (a), it can be observed that when there are a few SN in the network and a few SN transmitting in the CFP, the RTT for the SN in the SCP is low and stable, being even lower than the RTT for the SN in the CFP, as explained before. Figure 5 (b) illustrates how, in a network with a few SN, the communications performance of the SN transmitting in the SCP degrades when the number of SN transmitting in the CFP goes close to the maximum. It can be observed that the median of the RTT for the SN transmitting in the SCP is now higher than for the SN in the CFP, that such median values are not so stable and that the dispersion in the RTT is much higher (boxes are longer and outliers appear). Figure 5 (c) illustrates that such a degradation in the communications performance of the $\mathrm{SN}$ in the SCP when the $\mathrm{SN}$ in the CFP gets closer to the maximum is much worse when the total number of SN in the network increases up to 100 .

\section{CONClusions}

The paper presents the implementation for the Contention Free Period (CFP) carried out in the PRIME network simulator SimPRIME [11], as defined in the the ITU-T G.9904 standard [7]. In addition to this, based on this implementation, the use of the CFP is assessed from the point of view of the overall network performance and of the quality of service for the individual nodes.

As a general conclusion, the answer to the question in the title of the paper would be that the overall performance of PRIME networks is better when all the Service Nodes transmit together using the SCP, specially when the total number of Service Nodes is high. Nevertheless, the simulations carried out in this study also proves that the use of the CFP can guarantee communications with low jitter and stable RoundTrip Times (RTT) for a given group of Service Nodes and that, if such a group is not that big, which depends indeed on the size of the network, the overall performance of the network is not compromised. This fact makes CFP an interesting feature for, e.g.: (1) communicating with specific and highly sensible meters present among the overall low-voltage network (e.g., a subset of meters whose measurements are enough to estimate the state of the low voltage network); (2) controlling distributed energy resources (using CFP) in parallel with the metering traffic (using SCP) using the same PRIME network.

Additionally, according to the analysis carried out in the paper, it can be concluded that the standard does not allow for a high number of Service Nodes using the CFP. That is a consequence of the super-frame structure imposed in PRIME networks. To loosen this limit, and increase the advantages that this mechanism provides, Service Nodes could be organized in a way that they take turns in different super-frames to transmit using the CFP. For instance, half of them could use odd superframes and the other half could use even ones. This solution 


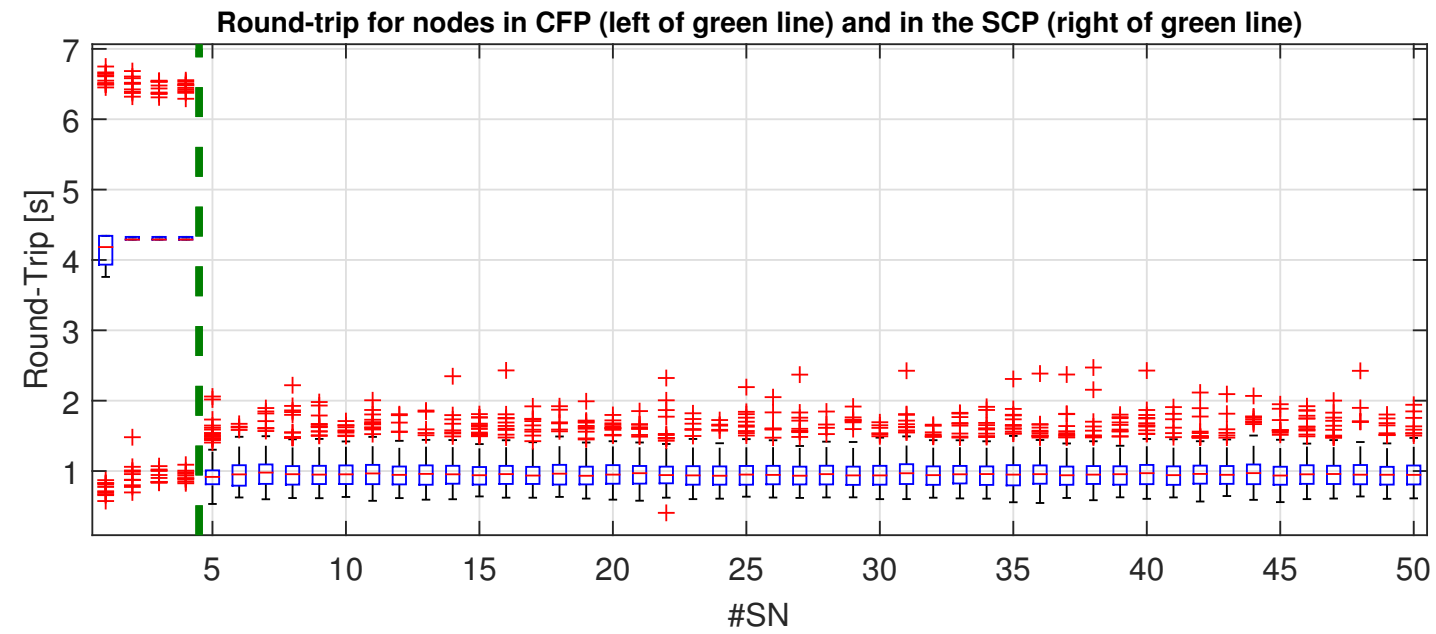

(a) 50 service nodes in total. 4 transmitting on CFP, the rest on SCP.

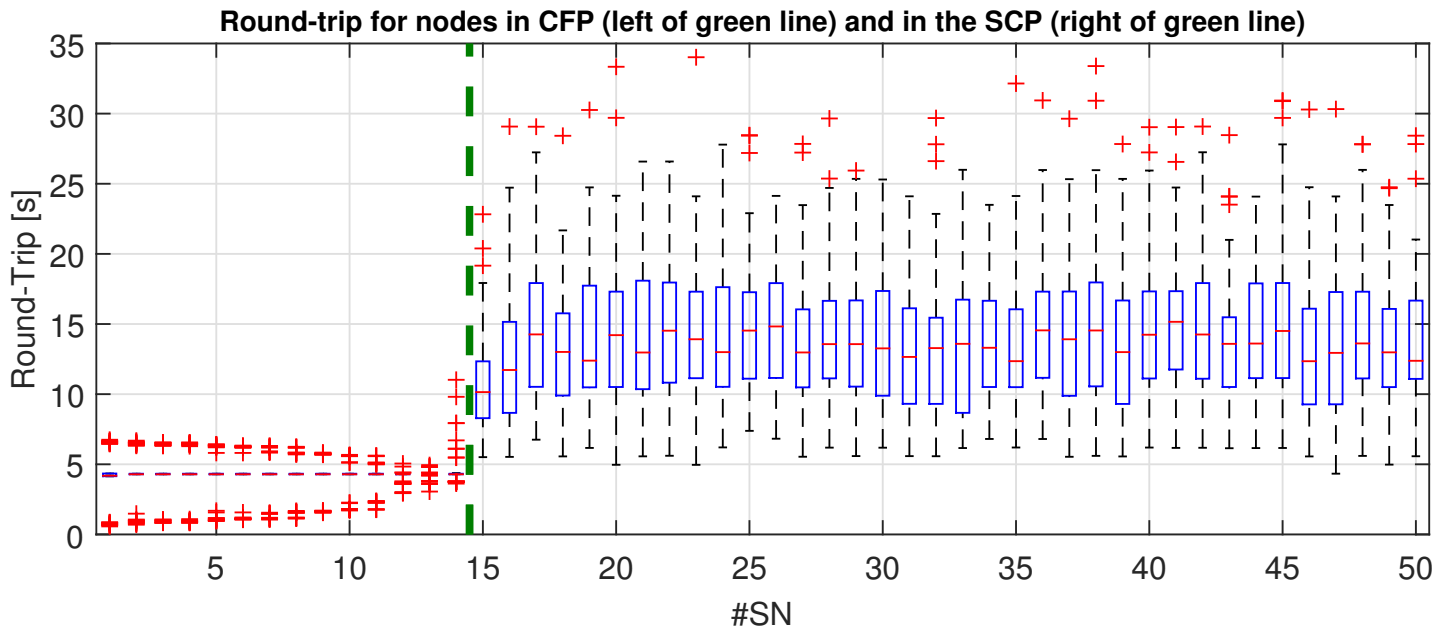

(b) 50 service nodes in total. 14 transmitting on CFP, the rest on SCP.

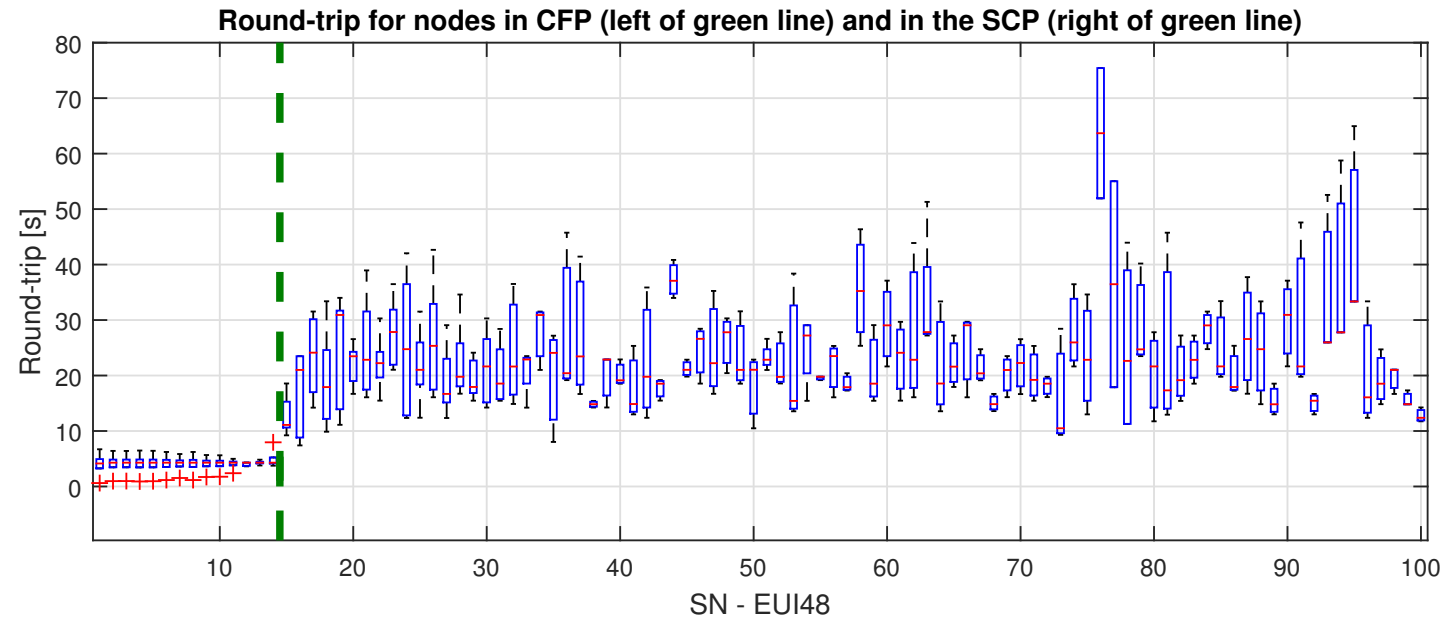

(c) 100 service nodes in total. 14 transmitting on CFP, the rest on SCP.

Fig. 6: RTT per node for different number of SN on CFP and SCP. 
would come at the cost of increasing the RTT guaranteed to the Service Nodes in the CFP.

To sum up, the use of the CFP could provide very interesting features to devices installed in PRIME-compliant LV networks running applications that require a certain QoS. This MAC mechanism is not being currently exploited in already deployed networks since they are used for applications which do not present very challenging communications constraint. However, it will be important in the near feature in order to make the most out of the already deployed infrastructures. Therefore, its achievable performance and dependencies need to be further investigated in more different scenarios (e.g., urban, dense rural, disperse rural). In addition, physical layers effects, such as noise, should be also considered, beside the overall number of $\mathrm{SN}$ and the number of $\mathrm{SN}$ in the CFP, since this kind of parameters may have a remarkable impact on the results of the simulations.

\section{ACKNOWLEDGMENT}

The research leading to these results has been partly funded by the Spanish Ministry of Economy and Competitiveness through the network of excellence REDYD2050 (ENE2015-70032-REDT). The authors would like to thank the REDYD2050 members. As a kind of minor tribute, the authors would like to dedicate the paper to the memory of Prof. Dr. Sadot Alexandres, who passed away during its preparation.

\section{REFERENCES}

[1] N. Uribe-Pérez, L. Hernández, D. de la Vega, and I. Angulo, "State of the Art and Trends Review of Smart Metering in Electricity Grids," Applied Sciences, vol. 6, no. 3, pp. 1-24, 2016.

[2] N. Andreadou, M. Guardiola, and G. Fulli, "Telecommunication Technologies for Smart Grid Projects with Focus on Smart Metering Applications," Energies, vol. 9, no. 5, p. 375, 2016.

[3] E. Commission, "Cost-benefit analyses and state of play of smart metering deployment in the EU-27." Tech. Rep., 2014.

[4] G. López, J. I. Moreno, H. Amarís, and F. Salazar, "Paving the road toward Smart Grids through large-scale advanced metering infrastructures," Electric Power Systems Research, vol. 120, pp. 194-205, 2015.

[5] C. Cano, A. Pittolo, D. Malone, L. Lampe, A. M. Tonello, and A. G. Dabak, "State of the art in power line communications: From the applications to the medium," IEEE Journal on Selected Areas in Communications, vol. 34, no. 7, pp. 1935-1952, July 2016.

[6] "PRIME Alliance Official Web Site." [Online]. Available: http://www.prime-alliance.org/

[7] ITU-T, "G.9904 : Narrowband orthogonal frequency division multiplexing power line communication transceivers for PRIME networks." Tech. Rep., 2012.

[8] A. Sendin, I. Urrutia, M. Garai, T. Arzuaga, and N. Uribe, "Narrowband PLC for LV smart grid services, beyond Smart Metering," 18th IEEE International Symposium on Power Line Communications and Its Applications, pp. 168-172, mar 2014.

[9] J. Matanza, S. Kiliccote, S. Alexandres, and C. Rodrguez-Morcillo, "Simulation of low-voltage narrow-band power line communication networks to propagate openadr signals," Journal of Communications and Networks, vol. 17, no. 6, pp. 656-664, Dec 2015.

[10] N. Uribe, I. Angulo, D. De la Vega, A. Sendin, I. Urrutia, L. Marron, and A. Arrinda, "TCP/IP Capabilities over NB-PLC for Smart Grid Applications: Field Validation," 20th IEEE International Symposium on Power Line Communications and Its Applications, april 2017.

[11] J. Matanza, "PRIME Network Simulator - SimPRIME." [Online]. Available: https://www.iit.comillas.edu/technology-offer/simprime
[12] J. Matanza Domingo, "Improvements in the PLC Systems for Smart Grids Environments," Ph.D. dissertation, Pontifical University of Comillas, 2013.

[13] E. Alonso, J. Matanza, C. Rodriguez-Morcillo, and S. Alexandres, "A switch promotion algorithm for improving prime plc network latency," in 18th IEEE International Symposium on Power Line Communications and Its Applications, March 2014, pp. 278-283.

[14] E. Alonso, J. Matanza, and C. Rodriguez-Morcillo, "Performance evaluation of AMR simultaneous polling strategies in a PRIME PLC network," 2015, pp. 101-106.

[15] OMNeT++ Community, "OMNeT++." [Online]. Available: http://www.omnetpp.org/

[16] J. Matanza, S. Alexandres, and C. Rodriguez-Morcillo, "Advanced metering infrastructure performance using european low-voltage power line communication networks," IET Communications, vol. 8, no. 7, pp. 1041-1047, May 2014.

[17] M. Seijo, G. Lopez, J. Matanza, and J. I. Moreno, "Planning and performance challenges in power line communications networks for smart grids," Int. J. Distrib. Sen. Netw., vol. 2016, pp. 28-, Mar. 2016.

[18] L. Gonzalez-Sotres, C. Mateo, P. Frias, C. Rodriguez-Morcillo, and J. Matanza, "Replicability analysis of plc prime networks for smart metering applications," IEEE Transactions on Smart Grid, vol. PP, no. 99 , pp. 1-1, 2016

[19] M. Seijo, G. Lopez, J. I. Moreno, J. Matanza, S. Alexandres, C. Rodriguez-Morcillo, and F. Martin, "Let there be light: Dissecting how PRIME networks work based on actual traffic traces," 2015 IEEE International Conference on Smart Grid Communications, SmartGridComm 2015, pp. 472-477, 2016. 